\title{
Bounds on the Achievable Region for Certain Multiple Description Coding Problems ${ }^{\dagger}$
}

\author{
Raman Venkataramani \\ Univ. of Illinois at \\ Urbana-Champaign \\ Urbana, IL 61801, U.S.A. \\ raman@ifp.uiuc.edu
}

\author{
Gerhard Kramer \\ Bell Labs, Lucent Technologies \\ 600 Mountain Avenue \\ Murray Hill, NJ 07974, U.S.A. \\ gkr@bell-labs.com
}

\author{
Vivek K Goyal \\ Digital Fountain \\ 39141 Civic Center Drive \\ Fremont, CA 94538, U.S.A. \\ v.goyal@ieee.org
}

\begin{abstract}
An achievable region for the $L$-channel multiple description coding problem is presented. This region generalizes previous two-channel results of El Gamal and Cover and of Zhang and Berger. New outer bounds on the rate distortion region for memoryless Gaussian sources with mean-squared error distortion are also derived. For the Gaussian source, the achievable region meets the outer bound at certain points.
\end{abstract}

\section{Problem Description}

Consider a source that emits a sequence $X^{N}=$ $X^{(1)}, X^{(2)}, \ldots, X^{(N)}$ of $N$ independent and identically distributed (i.i.d.) random variables. $X^{N}$ is encoded into $L$ descriptions $J_{1}, J_{2}, \ldots, J_{L}$ at rates $R_{1}, R_{2}, \ldots, R_{L}$ nats per source symbol. Suppose that each description is either transmitted error-free or lost completely. Thus the receiver encounters one of $2^{L}$ configurations depending on which descriptions are received. Excepting the trivial case where no description is received, we can represent the receiver as a collection of $2^{L}-1$ decoders, where each decoder produces an output based on a non-empty subset of $\left\{J_{1}, \ldots, J_{L}\right\}$.

Let $\mathcal{L}=\{1, \ldots, L\}$ and let $2^{\mathcal{L}}$ be its power set. For every $\mathcal{K} \in 2^{\mathcal{L}}$, let $X_{\mathcal{K}}^{N}=X_{\mathcal{K}}^{(1)}, \ldots, X_{\mathcal{K}}^{(N)}$ denote the output of the decoder whose inputs are $\left\{J_{k}: k \in \mathcal{K}\right\}$. Next let $d_{\mathcal{K}}=E\left[\frac{1}{N} \sum_{n=1}^{N} \delta_{\mathcal{K}}\left(X^{(n)}, X_{\mathcal{K}}^{(n)}\right)\right]$ denote the expected distortion per source symbol associated with the output $X_{\mathcal{K}}^{N}$, where $\delta_{\mathcal{K}}(\cdot, \cdot)$ is a distortion measure. Our problem is to find the set of rates $\left\{R_{1}, \ldots, R_{L}\right\}$ and distortions $\left\{d_{\mathcal{K}}: \mathcal{K} \in 2^{\mathcal{L}}-\{\emptyset\}\right\}$ that are achievable in the usual Shannon sense. We call this region the rate-distortion $(R D)$ region.

\section{An Achievable Region}

The set difference between collections of sets $\mathcal{C}$ and $\mathcal{D}$ is denoted $\mathcal{C}-\mathcal{D}=\{\mathcal{M} \in \mathcal{C}: \mathcal{M} \notin \mathcal{D}\}$. Also, we write $R_{\mathcal{K}}$ as a shorthand for $\sum_{k \in \mathcal{K}} R_{k}$ and $X_{(\mathcal{C})}$ for a collection of random variables $\left\{X_{\mathcal{N}}: \mathcal{N} \in \mathcal{C}\right\}$. Our first result is an achievable region for the general $L$-description problem.

Theorem 1 Let $X_{\left(2^{\mathcal{L}}\right)}$ be $2^{L}$ finite-alphabet random variables jointly distributed with $X$. Then the $R D$ region contains the rates and distortions satisfying

$$
\begin{aligned}
d_{\mathcal{K}} \geq & E \delta_{\mathcal{K}}\left(X, X_{\mathcal{K}}\right) \\
R_{\mathcal{K}} \geq & (|\mathcal{K}|-1) I\left(X ; X_{\emptyset}\right)-H\left(X_{\left(2^{\mathcal{K}}\right)} \mid X\right) \\
& \quad+\sum_{\mathcal{M} \subseteq \mathcal{K}} H\left(X_{\mathcal{M}} \mid X_{(2 \mathcal{M}}-\{\mathcal{M}\}\right)
\end{aligned}
$$

for every $\mathcal{K} \in 2^{\mathcal{L}}-\{\emptyset\}$, where $|\mathcal{K}|$ is the cardinality of $\mathcal{K}$.
In Theorem $1, X_{\emptyset}$ is an arbitrary random variable. For $L=$ 2 , this result generalizes the result of Zhang and Berger [1]. Additionally, with $X_{\emptyset}$ set to a constant, e.g. 0, it reduces to the result of El Gamal and Cover [2].

Theorem 1 holds more generally for well-behaved continuous sources if all entropies $H(\cdot)$ are replaced by differential entropies $h(\cdot)$. We next focus exclusively on the Gaussian source with mean-squared error distortion.

\section{The Quadratic Gaussian Case}

The following outer bound generalizes a result of Ozarow [3]. For this result, a collection of $M$ disjoint sets $\left\{\mathcal{K}_{m}\right\}_{m=1}^{M}$ is called a partition of a set $\mathcal{K}$ if $\bigcup_{m=1}^{M} \mathcal{K}_{m}=\mathcal{K}$.

Theorem 2 For each $\mathcal{K} \in 2^{\mathcal{L}}$, the achievable rates $R_{\ell}, \ell \in \mathcal{L}$, and distortions $d_{\mathcal{K}}, \mathcal{K} \in 2^{\mathcal{L}}$, satisfy

$$
e^{-2 R_{\mathcal{K}}} \leq \min _{\left\{\mathcal{K}_{m}\right\}_{m=1}^{M}} \inf _{\lambda \geq 0}\left(d_{\mathcal{K}} \frac{\prod_{m=1}^{M}\left(d_{\mathcal{K}_{m}}+\lambda\right)}{\left(d_{\mathcal{K}}+\lambda\right)(1+\lambda)^{M-1}}\right)
$$

where the minimization is performed over all partitions of $\mathcal{K}$.

Example Let the source emit unit-variance i.i.d. Gaussian random variables, and suppose that we care only about the reconstructions from individual descriptions $X_{\{1\}}, X_{\{2\}}, \ldots, X_{\{L\}}$, and the reconstruction from all the descriptions $X_{\mathcal{L}}$. An important operating point is that of equal side distortions and rates on all channels: $d_{\{\ell\}}=d$ and $R_{\ell}=R \geq-\frac{1}{2} \log d, \ell \in \mathcal{L}$. For this operating point, we can show using Theorems 1 and 2 , that the best central distortion achievable is

$$
d_{\mathcal{L}}=\sup _{\lambda \geq 0}\left(\frac{e^{-2 L R} \lambda(1+\lambda)^{L-1}}{(d+\lambda)^{L}-e^{-2 L R}(1+\lambda)^{L-1}}\right) .
$$

In fact, we can show that the outer and inner bounds meet everywhere on an open set in the vicinity of this point.

\section{REFERENCES}

[1] Z. Zhang and T. Berger, "New results in binary multiple descriptions," IEEE Trans. Inform. Th., vol. IT-33, no. 4, pp. 502-521, July 1987.

[2] A. A. El Gamal and T. M. Cover, "Achievable rates for multiple descriptions," IEEE Trans. Inform. Th., vol. IT-28, no. 6, pp. 851-857, November 1982.

[3] L. Ozarow, "On a source coding problem with two channels and three receivers," Bell System Tech. J., vol. 59, no. 10, pp. 1909-1921, December 1980.

\footnotetext{
${ }^{\dagger}$ This work was completed at Bell Labs, Lucent Technologies.
} 